\title{
Scale-free network growth by ranking
}

\author{
Santo Fortunato, ${ }^{1,2}$ Alessandro Flammini, ${ }^{1}$ and Filippo Menczer ${ }^{1}$ \\ ${ }^{1}$ School of Informatics, Indiana University, Bloomington, IN 47406, USA \\ ${ }^{2}$ Fakultät für Physik, Universität Bielefeld, D-33501 Bielefeld, Germany
}

\begin{abstract}
Network growth is currently explained through mechanisms that rely on node prestige measures, such as degree or fitness. In many real networks those who create and connect nodes do not know the prestige values of existing nodes, but only their ranking by prestige. We propose a criterion of network growth that explicitly relies on the ranking of the nodes according to any prestige measure, be it topological or not. The resulting network has a scale-free degree distribution when the probability to link a target node is any power law function of its rank, even when one has only partial information of node ranks. Our criterion may explain the frequency and robustness of scale-free degree distributions in real networks, as illustrated by the special case of the Web graph.
\end{abstract}

PACS numbers: 89.75.-k, 89.75.Hc

Keywords: Complex networks, rank, degree distribution.

Scholars have become interested in the many complex networks with long-tailed degree distribution [1, 2, 3] due to their peculiar structural features like resilience [4] and to the critical dynamical processes taking place on these networks, including epidemics spreading [5], search 6], and opinion formation [7]. The most popular explanation for the origin of scale-free networks is preferential attachment [8], according to which a newly created node is connected to a pre-existing one with a probability exactly proportional to the number of links (degree) of the target node. This mechanism embodies the intuitive idea of a 'rich get richer' dynamics. In the limit of infinitely many nodes, the degree distribution of the resulting network has a power law tail with exponent $\gamma=3$. Preferential attachment has been explicitly or implicitly embodied in many successive models of network growth [9, 10].

Krapivsky and Redner [1] showed that the proportionality between linking probability and target node degree is a necessary ingredient of preferential attachment; if the linking probability is a power of the degree with exponent $\alpha$, the resulting network has a power law degree distribution only when $\alpha=1$. For $\alpha<1$ the degree distribution is a power law multiplied by a stretched exponential and for larger values of $\alpha$ the model yields star-like networks. This seems at odds with the abundance and robustness of scale-free degree distributions in real networks. Other proposed mechanisms do not rely on preferential attachment. If the attraction of links depends on some "fitness" property of the target node, the networks display scale-free degree distributions for some suitable choices of the fitness distribution [12, 13].

Whether the link attractiveness of a node depends on a prestige measure exogenous or endogenous to the network topology, this information may not be available in real cases. In a social network, for example, we could assume that the probability for a person to make new friends is proportional to properties such as popularity, attractiveness, or wealth - all typically difficult for strangers to quantify, measure, or discover.
While the absolute importance of an object is often unknown, it is quite common to have a clear idea about the relative values of two objects. One can often say who is the richer or more popular between two individuals. As another example, search engine users only see how Web pages are ranked. The perception of how items are ranked requires far less information than their actual importance. Here we propose a model of network growth that focuses on the relative rather than absolute importance of the nodes, which are ranked according to an arbitrary prestige measure. We show that scale-free networks emerge for a very general form of the linking probability and are stable for a large range of the parameters describing the growth. The result holds even if new nodes have information on only subsets of older nodes.

First, a prestige ranking criterion is selected. At the $(t+1)$-th iteration, the new node $t+1$ is created and new links are set from it to $m$ pre-existing nodes. The previous $t$ nodes are ranked according to prestige, and the linking probability $p(t+1 \rightarrow j)$ that node $t+1$ be connected to node $j$ only depends on the rank $R_{j}$ of $j$ :

$$
p(t+1 \rightarrow j)=\frac{R_{j}^{-\alpha}}{\sum_{k=1}^{t} R_{k}^{-\alpha}}
$$

where $\alpha>0$ is a real-valued parameter. The linking probability clearly decreases with increasing rank.

The choice of prestige measure is arbitrary. We discuss both topological measures (age $t$ and degree $k$ ) and exogenous ones (any node fitness $\eta$ ).

If the nodes are sorted by age, from the oldest to the newest, the label of each node coincides with its rank, i.e. $R_{t}=t \forall t$. In this special case, our linking probability coincides with that of the so-called static network model [14]. We can calculate the number of links that the $R$-th node will attract since its creation. Suppose that the evolution of the network stops when $N$ nodes are created. At each iteration a constant number $m$ of links are created between the new node and the older ones. The expected total number $k_{R}^{N}$ of links that the 


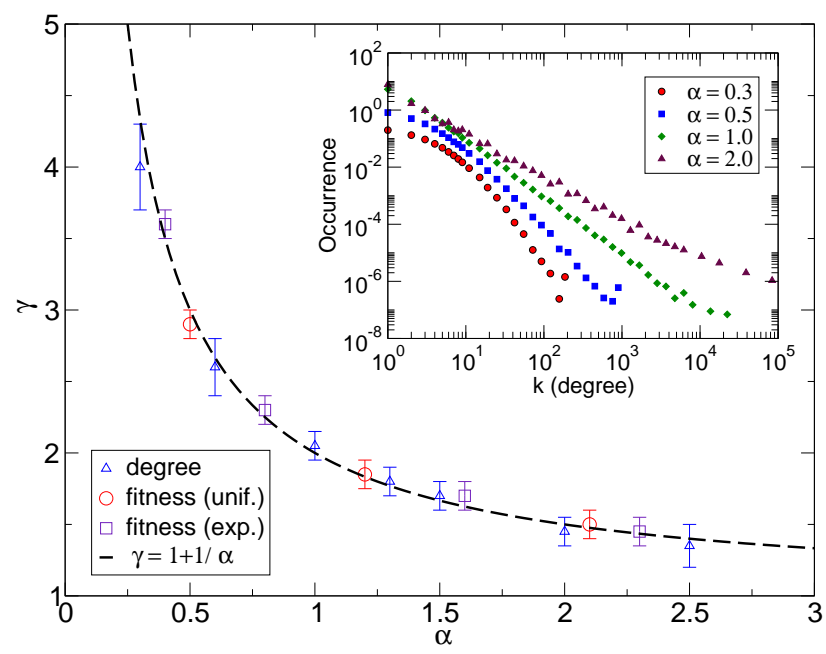

FIG. 1: Inset: In-degree distributions for networks built according to our model (ranking by degree). The number of nodes is $N=10^{6}$. Data are averaged within logarithmic bins of degree and shifted along the $y$-axis for illustration purposes. The main curve plots the degree distribution exponent $\gamma$ as a function of $\alpha$, from simulations with nodes ranked by various criteria. Error bars represent standard errors on the best fit estimates of $\gamma$, while the dashed line is the prediction of Eq. 4.

$R$-th node has attracted at the end is

$$
k_{R}^{N}=\sum_{t=R+1}^{N} \frac{m R^{-\alpha}}{\sum_{j=1}^{t} j^{-\alpha}} .
$$

The first sum runs over $N-R$ terms because $N-R$ nodes are created after node $R$, and each of them can be connected to $R$. We now approximate the sums with integrals and assume that $N \gg R$, as we are ultimately interested in the thermodynamic limit. We find that $k_{R}^{N}=A R^{-\alpha}$, where $A$ is a function of $N, \alpha$ and $m$. Knowing $k_{R}^{N}$ it is possible to find how many nodes $N_{k}$ have the same expected number of links $k$. The ratio $N_{k} / N$ in the limit of large $N$ yields the probability $p(k, N)$ that a node of the network has degree $k$ :

$$
p(k, N) \sim k^{-(1+1 / \alpha)} .
$$

Eq. 3 shows that the degree distribution of the network follows a power law with exponent

$$
\gamma=1+1 / \alpha
$$

for any value of $\alpha$. Since $\gamma$ can take any value greater than 1 , we can in principle reproduce the exponents measured in real systems. For $\alpha>1(\gamma<2)$ a few nodes attract a finite fraction of all links (condensation); in the limit case in which the power law of Eq. 1 is replaced by a simple exponential the network still has a long-tailed degree distribution with $\gamma=1$ (as in the limit $\alpha \rightarrow \infty$ ).

Let us now consider a more realistic ranking criterion, the in-degree. The number of incoming links of a node represents how many times the node has been selected by its peers. For undirected networks we can equivalently use the degree. Nodes with (in-)degree zero, which if present are a problem for the extension of other growth models to directed networks, do not raise an issue here because they have ranks expressed by positive numbers, like all other nodes.

To see what kind of networks emerge with this new prestige measure, we cannot apply the above derivation because the degree-based ranking of a node can change over time. On the other hand, for a growing network there is a strong correlation between the age of a node and its degree, as older nodes have more chances to receive links. Furthermore the ranking of nodes according to degree is quite stable [15]. Therefore we expect the same result as for the ranking by age.

To verify our expectation we performed Monte Carlo simulations of the network growth process with the new degree-based ranking strategy. The inset of Fig. 11 shows the degree distributions of four networks, corresponding to various values of the exponent $\alpha$. In the logarithmic scale of the plot the tails appear as straight lines, as one would expect for scale-free distributions. To verify that the relation between $\alpha$ and the exponent $\gamma$ of the degree distribution is the one predicted by our model, in the main plot of Fig. 11 we compare various pairs $(\alpha, \gamma)$ with the hyperbola of Eq. 4 The agreement is evident.

A striking feature of our model is that it generates scale-free networks even when ranking nodes by a prestige measure unrelated to the network topology, i.e. by some exogenous fitness attribute of the nodes. Suppose we assign a fitness $\eta$ to the nodes according to an arbitrary distribution. Let $R_{j}(t)$ be the expected rank of node $j$, with fitness $\eta_{j}$, among $t$ nodes. As $R_{j}(t)$ is asymptotically proportional to $t$, we can write $R_{j}(t)=t \rho\left(\eta_{j}\right)$, where the relative rank $\rho\left(\eta_{j}\right)$ depends only on $\eta_{j}$. By replacing the rank in the linking probability of Eq. I by the expression $t \rho\left(\eta_{j}\right)$, we can factor out $t^{-\alpha}$ from both the numerator and the denominator, leading us back to the case of static ranking discussed above.

Monte Carlo simulations confirm the result. We used uniform, exponential and power law fitness distributions. The resulting networks have degree distributions with power law tails, for any value of $\alpha$ and any fitness distribution. The relation between the exponents $\gamma$ and $\alpha$ is in agreement with the prediction of Eq. 4 in all cases. In Fig. 1 we illustrate this relation for the uniform and exponential cases.

Most models of network growth assume that a new node can be linked to any existing node, chosen according to some criterion. This requires that the new node be aware of the status of all its peers. Such an assumption of complete knowledge of the network may not be realistic. For instance, in a large social network nobody knows everybody else. It is reasonable to suppose that the knowledge that each node has of the network is lim- 
ited to a sample of nodes, which is in general different from node to node. In the previous example, each person has his/her own group of acquaintances. Having access only to portions of a network can profoundly affect the dynamics of network growth. Mossa et al. [16] showed that preferential attachment would yield power law degree distributions ending with exponential cutoffs.

Let us check whether and to which extent the hypothesis of limited information affects the dynamics of the rank-driven model proposed here. We assume that whenever a new node is created, it can 'see' each of the preexisting nodes with a probability $h$. We shall see that if $h$ is constant, our earlier result holds; if $h$ is power-law distributed, the scenario is more complex, but one recovers long-tailed degree distributions in most cases.

If $h$ is constant the size distribution of the subsets accessed by the new nodes is a binomial peaked at $h t$, where $t$ is the number of nodes of the network after $t$ iterations. This means that most subsets will have a size of about $h t$. Let us assume that the nodes are ranked according to their age, making a formal analysis possible. The linking probability is still given by Eq. 1 with the important caveat that now we deal only with the nodes within the subset accessed by the newly created node. So the ranks of Eq. प 1 refer to the ordering of the nodes of the subset, not of all nodes like before. Let us indicate the 'local' ranks with $r$, to distinguish them from the global ranks $R$ we have dealt with so far.

When a new node $t+1$ is created, it knows a list of $n$ older nodes. One can calculate the probability $p(R, r, t, n, h)$ that node $R$ has local rank $r$ within this list, and from this the probability $p(t+1 \rightarrow R, r, n, h)$ that such a node be linked to $t+1$. Then we sum over the possible ranks $r$ of node $R$ in the list $(r \in 1 \ldots n)$ and all possible subset sizes $(n \in 1 \ldots t)$. The result yields the linking probability $p(t+1 \rightarrow R, h)$ of $t+1$ to $R$ :

$$
\begin{aligned}
& p(t+1 \rightarrow R, h)= \\
& \sum_{n=1}^{t} \sum_{r=1}^{n} \frac{h^{n}(1-h)^{t-n} r^{-\alpha}}{\sum_{m=1}^{n} m^{-\alpha}}\left(\begin{array}{c}
R-1 \\
r-1
\end{array}\right)\left(\begin{array}{c}
t-R \\
n-r
\end{array}\right) .
\end{aligned}
$$

From Eq. 5 we see that if $h=1$, which corresponds to a list with all $t$ nodes, one recovers Eq. 1 as expected. For $h<1$, however, it is not possible to derive a close expression for $p(t+1 \rightarrow R, h)$, so we performed Monte Carlo simulations of the process leading to Eq. 5 In every simulation we produced a large number of lists, each formed by sampling nodes with probability $h$. At the beginning of the simulation we initialized all entries of the array $p(t+1 \rightarrow R, h)=0$. Once a list was completed, we added to the entries of $p(t+1 \rightarrow R, h)$, corresponding to the nodes of the list, the linking probability as given by Eq. 10 (with the proper normalization). With this method we simulated systems with up to $N=10^{6}$ nodes.

For $t$ not too small, $p(t+1 \rightarrow R, h)$ is well approxi- mated by the following function:

$$
p(t+1 \rightarrow R, h) \sim\left\{\begin{array}{l}
\frac{(\alpha-1) h^{\alpha}}{\alpha h^{\alpha-1}-t^{1-\alpha}} \text { if } 1 \leq R \leq\left[\frac{1}{h}\right] \\
\frac{(\alpha-1) R^{-\alpha}}{\alpha h^{\alpha-1}-t^{1-\alpha}} \text { if }\left[\frac{1}{h}\right] \leq R \leq t .
\end{array}\right.
$$

The first $\left[\frac{1}{h}\right]$ nodes have the same probability of being selected. For the other nodes, $p(t+1 \rightarrow R, h)$ has the same dependence on $R$ as in the case in which there is complete information on the network $(h=1)$. This means that in a network grown with the linking probability of Eq. first $\left[\frac{1}{h}\right]$ nodes will have approximately the same number of links, whereas the degrees of the others will have the distribution of Eq. 3 If $h$ is independent of $N$, the subset of equiprobable nodes does not grow with $N$ and has no structural relevance when $N \rightarrow \infty$; if $h \sim 1 / N$ the subset of equiprobable nodes is a fraction of the network and the degree distribution has an exponential cutoff. Monte Carlo simulations confirmed the result. We conclude that the degree distribution of networks grown with our ranking strategy is the same whether new nodes have access to the full network or just to subsets of it, as long as the subsets contain a constant proportion of the network nodes. The latter assumption may not be realistic, as the number of contacts may vary appreciably from node to node. Next we extend our analysis to this case.

In general, if $h$ is distributed according to a function $S(h)$, we need to convolute the $p(t+1 \rightarrow R, h)$ of Eq. 囵 with $S(h)$ to get the linking probability $p_{S}(t+1 \rightarrow R)$ of the full process,

$$
p_{S}(t+1 \rightarrow R)=\int_{h_{m}}^{h_{M}} S(h) p(t+1 \rightarrow R, h) d h,
$$

where $h_{m}$ and $h_{M}$ are the extremes of the interval where $S(h)$ is defined. We consider the following simple probability distribution for $h$ :

$$
S(h)=\frac{\beta-1}{t^{\beta-1}-1} h^{-\beta}
$$

where $\beta>0$. The function is defined in the range $h \in[1 / t, 1]$. In fact, for a network with $t$ nodes, in order to have at least one item in a random selection of nodes one needs a probability $h \geq 1 / t$. The prefactor ensures the normalization of the function in the interval $[1 / t, 1]$. As we discuss later, the choice of the power law in Eq. [ is a realistic one; it also accounts for the two limit cases of uniform $(\beta=0)$ and exponential $(\beta \rightarrow \infty)$ distributions; finally it allows us to treat the problem analytically, provided we introduce reasonable approximations.

We plug Eqs. [6] and 8 into Eq. 7 With $R$ fixed, we split the integral over the two $h$ domains corresponding to the regimes of Eq. 6. Depending on the values of the parameters $\alpha$ and $\beta$, we can neglect different terms in the resulting integrands, leading to four cases for the asymptotic dependence of the linking probability on rank.

In three of the cases $p_{S}(t+1 \rightarrow R)$ has a power law dependence on $R$ : the networks grown for the corresponding values of $\alpha$ and $\beta$ will then have scale-free degree 


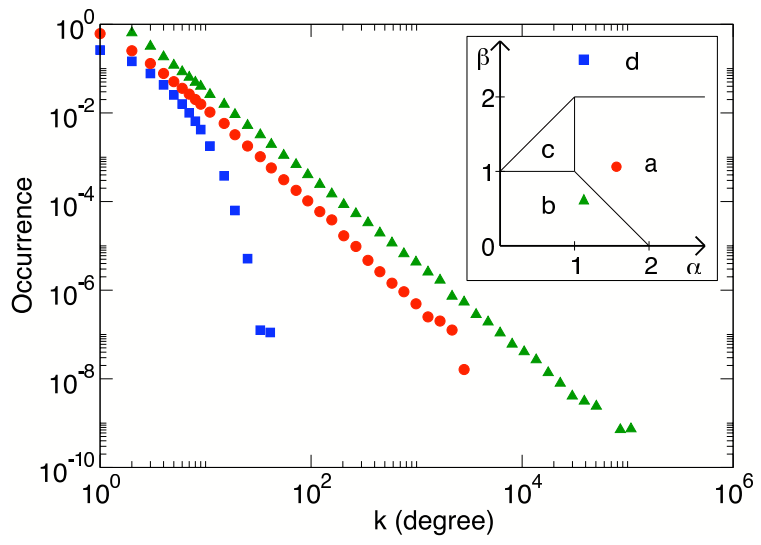

FIG. 2: Degree distributions for networks grown according to the proposed model, with incomplete information. Inset: Regions of the parameter space for the four regime cases. The marked points correspond to the $(\alpha, \beta)$ values that generate the distributions in the main plot.

distributions. The power law distribution of the probability $h$ affects the value of the exponent $\gamma$ of the degree distribution, which no longer depends only on $\alpha$ and in one case is only a function of $\beta$. In the last case the linking probability is independent of the rank, so all nodes have the same chance of being linked and the degree distribution is exponential. The four cases correspond to regions of the $(\alpha, \beta)$ parameter quadrant (Fig. 2 inset). In cases $a, b$ and $c, p(k) \sim k^{-\gamma}$ with $\gamma_{a}=1+1 /(2-\beta)$, $\gamma_{b}=1+1 / \alpha$, and $\gamma_{c}=1+1 /(1+\alpha-\beta)$. In case $d, p(k)$ is exponential. Monte Carlo simulations confirm these predictions, as shown in Fig. 2 The results are identical if the nodes are ranked according to degree or fitness.

Compared to mechanisms proposed in the past to explain the emergence of scale-free networks, the rankbased model introduced here presents three main advantages. First, it assumes less information is available to nodes (or node creators); it seems more realistic in many real cases to imagine that the relative importance of items is easier to access than their absolute importance. Second, the link attractiveness of nodes is by no means restricted to topology; it can depend on exogenous attributes of the nodes, which makes our model suitable for applications in many different contexts. Third, the criterion is more robust in that: (i) it naturally extends to directed networks; (ii) it leads to long-tailed degree distributions for a broad class of linking probability functions - namely power laws of rank with any exponent $\alpha>0$, including the degenerate exponential case for $\alpha \rightarrow \infty$; and (iii) the scale-free degree distribution generated by the model is not affected by limiting the information available to subsets of nodes.

The rank-based model is directly applicable to the Web as a special case, if one considers the role of search engines in the discovery of pages [17]. When a user submits a query, the search engine ranks the results by various criteria including a topological prestige measure, PageRank, closely correlated with in-degree. Users do not know the PageRank of the search hits, but observe their ranking and thus are more likely to discover and link pages that are ranked near the top. By analyzing search engine logs one finds that (i) users click on result hits with a probability that is a power law function of rank matching Eq. 1] with $\alpha=1.6$; and (ii) user queries return hit sets whose size distribution matches the power law in Eq. 8 with $\beta=1.1$. Assuming that users tend to link pages that they discover by searching, and that they are only aware of the pages returned by search engines in response to their queries, our model predicts a scale-free distribution of in-degree with exponent $\gamma_{a}=2.1$ (cf. case $a$ and curve marked with circles in Fig. (2). This is in perfect agreement with established Web measurements 4 .

We thank A. Vespignani, M. Serrano, M. Boguñá, V. Colizza and M. Barthélemy for helpful discussions. Work funded in part by a Volkswagen Foundation grant to SF, NSF award 0348940 to FM, and Indiana University School of Informatics.

[1] S. N. Dorogovtsev and J. F. F. Mendes, Adv. in Phys. 51, 1079-1187 (2002).

[2] R. Albert and A.-L. Barabási, Rev. Mod. Phys. 74, 47-97 (2002).

[3] M. E. J. Newman, SIAM Review 45, 167-256 (2003).

[4] R. Albert, H. Jeong and A.-L. Barabási, Nature 406, 378-382 (2000).

[5] R. Pastor-Satorras and A. Vespignani, Phys. Rev. Lett. 86, 3200-3203 (2001).

[6] L. A. Adamic, R. M. Lukose, A. R. Puniyani and B. A. Huberman, Phys. Rev. E 64, 046135 (2001).

[7] S. Fortunato and D. Stauffer, Computer Simulations of Opinions and Their Reactions to Extreme Events, in Extreme Events in Nature and Society, eds. S. Albeverio et al. (Springer Verlag, Berlin-Heidelberg, 2005).

[8] A.-L. Barabási and R. Albert, Science 286, 509-512 (1999).

[9] J. Kleinberg, S. .R. Kumar, P. Raghavan, S. Rajagopalan and A. Tomkins, LNCS 1627, 1-18 (1999).

[10] S. N. Dorogovtsev, J. F. F. Mendes and A. N. Samukhin, Phys. Rev. Lett. 85, 4633-4636 (2000).

[11] P. L. Krapivsky, S. Redner and F. Leyvraz, Phys. Rev. Lett. 85, 4629-4632 (2000).

[12] G. Caldarelli, A. Capocci, P. De Los Rios and M. A. Muñoz, Phys. Rev. Lett. 89, 258702 (2002).

[13] M. Boguñá and R. Pastor-Satorras, Phys. Rev. E 68, 036112 (2003).

[14] K.-I. Goh, B. Kahng and D. Kim, Phys. Rev. Lett. 87, 278701 (2001).

[15] P. L. Krapivsky and S. Redner, Phys. Rev. Lett. 89, 258703 (2002).

[16] S. Mossa, M. Barthélemy, H. E. Stanley and L. A. N. Amaral, Phys. Rev. Lett. 88, 138701 (2002).

[17] S. Fortunato, A. Flammini, F. Menczer, A. Vespignani, The egalitarian effect of search engines, Tech. Rep. cs. CY/0511005 arXiv.org (2005). 